\title{
Discrimination of binary coherent states using a homodyne detector and a photon number resolving detector
}

\author{
Wittmann, Christoffer; Andersen, Ulrik Lund; Takeoka, Masahiro; Sych, Denis; Leuchs, Gerd
}

Published in:

Physical Review A

Link to article, DOI:

10.1103/PhysRevA.81.062338

Publication date:

2010

Document Version

Publisher's PDF, also known as Version of record

Link back to DTU Orbit

Citation (APA):

Wittmann, C., Andersen, U. L., Takeoka, M., Sych, D., \& Leuchs, G. (2010). Discrimination of binary coherent states using a homodyne detector and a photon number resolving detector. Physical Review A, 81(6), 062338. https://doi.org/10.1103/PhysRevA.81.062338

\section{General rights}

Copyright and moral rights for the publications made accessible in the public portal are retained by the authors and/or other copyright owners and it is a condition of accessing publications that users recognise and abide by the legal requirements associated with these rights.

- Users may download and print one copy of any publication from the public portal for the purpose of private study or research.

- You may not further distribute the material or use it for any profit-making activity or commercial gain

- You may freely distribute the URL identifying the publication in the public portal 


\title{
Discrimination of binary coherent states using a homodyne detector and a photon number resolving detector
}

\author{
Christoffer Wittmann, ${ }^{1,2}$ Ulrik L. Andersen, ${ }^{1,3}$ Masahiro Takeoka, ${ }^{4}$ Denis Sych, ${ }^{1,2}$ and Gerd Leuchs ${ }^{1,2}$ \\ ${ }^{1}$ Max Planck Institute for the Science of Light, Günther-Scharowsky-Straße 1, Bau 24, 91058 Erlangen, Germany \\ ${ }^{2}$ Institut für Optik, Information und Photonik, University of Erlangen-Nuremberg, Staudtstraße 7/B2, 91058 Erlangen, Germany \\ ${ }^{3}$ Department of Physics, Technical University of Denmark, Building 309, 2800 Kongens, Lyngby, Denmark \\ ${ }^{4}$ National Institute of Information and Communications Technology (NICT), 4-2-1 Nukui-kitamachi, Koganei, Tokyo 184-8795, Japan
}

(Received 13 April 2010; published 25 June 2010)

\begin{abstract}
We investigate quantum measurement strategies capable of discriminating two coherent states probabilistically with significantly smaller error probabilities than can be obtained using nonprobabilistic state discrimination. We apply a postselection strategy to the measurement data of a homodyne detector as well as a photon number resolving detector in order to lower the error probability. We compare the two different receivers with an optimal intermediate measurement scheme where the error rate is minimized for a fixed rate of inconclusive results. The photon number resolving (PNR) receiver is experimentally demonstrated and compared to an experimental realization of a homodyne receiver with postselection. In the comparison, it becomes clear that the performance of the PNR receiver surpasses the performance of the homodyne receiver, which we prove to be optimal within any Gaussian operations and conditional dynamics.
\end{abstract}

DOI: 10.1103/PhysRevA.81.062338

PACS number(s): 03.67.Hk, 03.65.Ta, 42.50.Lc

\section{INTRODUCTION}

In classical communication systems, information is usually encoded into orthogonal quantum or semiclassical states of light. An important example is binary phase shift keying with coherent states where the logical information, " 0 " and " 1 ," is encoded as two coherent states with large amplitudes and a relative phase of $\pi$. Since the two coherent states each possess a large amplitude (with opposite signs), they are nearly orthogonal and thus they can be easily discriminated using an interferometric measurement strategy. On the other hand, if the amplitude becomes very small, which is the case for quantum communication as well as long-distance amplification-free (e.g., free-space) classical communication, the two states are largely overlapping and thus nonorthogonal. Because of this nonorthogonality, the states can no longer be perfectly discriminated. Although perfect discrimination is not possible, it is of high interest to find optimized strategies in order to minimize measurement errors, thus keeping the error rate as low as possible and increasing the mutual information between sender and receiver. Moreover, the search for such optimized strategies are of utmost importance for many applications in quantum communication with quantum key distribution (QKD) being the prime example [1-3]. Finally, we note that the problem of finding optimized measurement schemes associated with a predefined alphabet is a fundamental problem in quantum mechanics $[4,5]$.

There are basically two well-known discrimination strategies. In the first strategy, all measurement outcomes are used (that is, it is deterministic) and, therefore, the resulting conclusions will be infected by errors. The idea is to optimize the strategy such that the probability for making an error is minimized. This strategy is known as minimum error state discrimination and has been analyzed by Helstrom [4]. The second discrimination strategy is probabilistic, and yields a valid outcome only when the conclusion drawn from the measurement is known to be error-free. Therefore, in this task the goal is to minimize the probability of inconclusive results (which are discarded). This strategy is known as unambiguous state discrimination (USD) and was originally proposed by Ivanovic, Dieks and Peres [5-8]. A combination of the two discrimination schemes where one allows for both erroneous and inconclusive results has also been treated theoretically. More precisely, the minimal probability of errors for a fixed probability of inconclusive results has been derived for pure and mixed states in Refs. [9] and [10], respectively.

For the discrimination of two coherent states with minimum error, several optimal and near-optimal receivers have been proposed [11-17]. Also, a device for implementing USD of coherent states was proposed by Huttner et al. [18] and later by Banaszek [19]. Some of these schemes have been experimentally accessed, such as the Dolinar receiver [20], the optimized displacement receiver [21,22], and a programmable receiver implementing USD [23]. However, the intermediate regime where errors as well as inconclusive results may occur has only recently been investigated experimentally [24].

In this paper, we elaborate on the work of Ref. [24]. We investigate two different receivers that belong to the intermediate regime. The first is a standard homodyne detector, and the second is a displacement-controlled photon number resolving detector [25]. In both receivers the measurement outcomes are postselected to obtain a specific relation between errors and inconclusive results. The postselection-based homodyne detector has been used in various protocols such as QKD [26-28], squeezed state and entangled state distillation [29-33], and quantum state engineering [34-37]. Here we thoroughly characterize the detector in terms of the discrimination between two coherent states. In addition we conduct a thorough experimental analysis of the displacement-based photon number resolving detector (PNR) that was introduced in Refs. [24,25]. We find that the displacement-based PNR receiver outperforms the standard homodyne detection.

The paper is organized as follows. First, we recapitulate the notion of intermediate measurement for coherent states in Sec. II. In Secs. III and IV, we consider measurements 
in the intermediate regime with two strategies: a receiver using a homodyne detector and a receiver using an optimized displacement combined with a photon number resolving detector. In Sec. III, we prove that the postselection-based homodyne scheme is the optimal strategy for realizing the intermediate measurement within all possible Gaussian operations and conditional dynamics. We demonstrate both receivers experimentally in Sec. V. Finally, we summarize the results in Sec. VII.

\section{INTERMEDIATE MEASUREMENT}

Let us assume a sender picks one signal state out of two pure and phase-shifted coherent states and sends it through a communication channel that preserves the quantum property of the state. On the other end of the channel, a receiver has to tell which state was chosen by the sender. Let us also assume that the a priori probabilities for the preparation of the states are $p_{1}=p_{2}=\frac{1}{2}$ and that the received states are $|-\alpha\rangle$ or $|+\alpha\rangle$.

The receiver measures the signal state and, based on the measurement outcome, guesses the state. Because of the nonorthogonality of the alphabet, however, the result will not be correct in all such attempts. In fact, the minimal error probability is given by the inner product of the states in the alphabet, $\sigma=|\langle-\alpha \mid \alpha\rangle|$. The maximally accessible information of the receiver is directly related to the minimal error rate.

The receiver can alternatively choose a measurement strategy that allows for inconclusive results. In this strategy, he will only accept states that are likely to be correctly identified, while he does not attempt to guess the results for signals associated with the inconclusive measurement results. This strategy is probabilistic as the outcomes are postselected. It can be shown that for higher probability of inconclusive results $p_{\text {inc }}$ (or lower acceptance probability $1-p_{\text {inc }}$ ) a lower error probability $p_{E}$ can be achieved.

This intermediate measurement strategy can be described by the three-component positive operator-valued measure (POVM) $\hat{\Pi}_{i}, i=1,2, ?$, where $\hat{\Pi}_{i}>0$ and $\hat{\Pi}_{1}+\hat{\Pi}_{2}+\hat{\Pi}_{?}=$ $\hat{I}$. Consequently, an inconclusive result will occur with the probability

$$
p_{\text {inc }}=p_{1}\left\langle-\alpha\left|\hat{\Pi}_{?}\right|-\alpha\right\rangle+p_{2}\left\langle\alpha\left|\hat{\Pi}_{?}\right| \alpha\right\rangle,
$$

where $\left\langle-\alpha\left|\hat{\Pi}_{?}\right|-\alpha\right\rangle\left(\left\langle\alpha\left|\hat{\Pi}_{?}\right| \alpha\right\rangle\right)$ represents the probability of inconclusive results when $|-\alpha\rangle(|\alpha\rangle)$ was prepared. Furthermore, the average error probability is given by

$$
p_{\mathrm{E}}=\frac{p_{1}\left\langle-\alpha\left|\hat{\Pi}_{2}\right|-\alpha\right\rangle+p_{2}\left\langle\alpha\left|\hat{\Pi}_{1}\right| \alpha\right\rangle}{1-p_{\text {inc }}},
$$

where $\left\langle-\alpha\left|\hat{\Pi}_{2}\right|-\alpha\right\rangle\left(\left\langle\alpha\left|\hat{\Pi}_{1}\right| \alpha\right\rangle\right)$ represents the error probability of mistakenly guessing $|\alpha\rangle(|+\alpha\rangle)$.

Finally the measurement strategy is optimized, such that the receiver's error probability is minimized for a given probability of inconclusive results. The error probability according to [9] is then given by

$$
p_{E} \geqslant \frac{1}{2}\left(1-\frac{\left[1-2 p_{\text {inc }}(1-\sigma)-\sigma^{2}\right]^{1 / 2}}{1-p_{\text {inc }}}\right),
$$

where the error rate is lower bounded by the inner product and the tolerated rate of inconclusive results. A receiver scheme achieving this optimal bound is yet unknown. In the following two sections, we investigate two near-optimal receivers: the postselection-based homodyne receiver and the displacementcontrolled photon number resolving detector.

\section{HOMODYNE RECEIVER}

A very simple receiver type, which is tunable in the probability of inconclusive results, is based on homodyne detection followed by postselection [26-28]. The schematic of this receiver is shown in Fig. 1(a). We now reconsider homodyne detection with postselection in the context of state discrimination in the aforementioned intermediate regime.

In the homodyne measurement, the local oscillator is set along the excitation of the coherent states resulting in a distribution of quadrature values as shown in Fig. 1(b). The conclusion of the receiver is deduced from the particular result, where positive measurement outcomes greater than a postselection threshold $B$ identify $|\alpha\rangle$ whereas negative outcomes less than the postselection threshold $-B$ identify $|-\alpha\rangle$. All measurement outcomes between the postselection thresholds are considered as inconclusive results. The corresponding POVMs are $\hat{\Pi}_{1}=\int_{-\infty}^{-B}|x\rangle\left\langle x\left|d x, \hat{\Pi}_{2}=\int_{B}^{\infty}\right| x\right\rangle\langle x| d x$, and $\hat{\Pi}_{?}=\hat{I}-\hat{\Pi}_{1}-\hat{\Pi}_{2}$, and result in the error probability

$$
p_{E, \mathrm{HD}}=\frac{1-\operatorname{erf}[\sqrt{2}(B+|\alpha|)]}{2\left(1-p_{\mathrm{inc}, \mathrm{HD}}\right)} .
$$

The probability of inconclusive results is found to be

$$
p_{\text {inc }, \mathrm{HD}}=\frac{1}{2}\{\operatorname{erf}[\sqrt{2}(B+|\alpha|)]+\operatorname{erf}[\sqrt{2}(B-|\alpha|)]\} .
$$

In the following, we prove that the postselected homodyne scheme is the optimal strategy for realizing the intermediate measurement within all possible Gaussian operations and conditional dynamics (classical feedback or feedforward). For simplicity we assume $\alpha$ is real and positive. Note that if the input alphabet as well as all operations are Gaussian, conditional dynamics is useless [38-40]. In our case, however, the input alphabet consists of an ensemble of two coherent states $p_{1}|-\alpha\rangle\left\langle-\alpha\left|+p_{2}\right| \alpha\right\rangle\langle\alpha|$. This is clearly non-Gaussian, and thus we cannot discard conditional dynamics as a tool to improve the discrimination task. We first briefly introduce the characteristic functional formalism of POVMs and then discuss the POVMs via Gaussian operations with and without conditional dynamics.

Here we use a characteristic function formalism similar to the approach used to prove the optimality of the homodyne measurement for discriminating binary coherent states with minimum error under Gaussian operations [17]. In quantum optics, the characteristic function $\chi(\omega)$ is often a useful tool to represent a continuous variable quantum state [41]. In an $N$-mode infinite-dimensional system, the characteristic function of a quantum state with the density matrix $\hat{\rho}$ is defined as

$$
\chi_{\rho}(\omega) \equiv \operatorname{Tr}\left[\hat{\rho} \exp \left(i \omega^{T} \hat{R}\right)\right]
$$


(a)

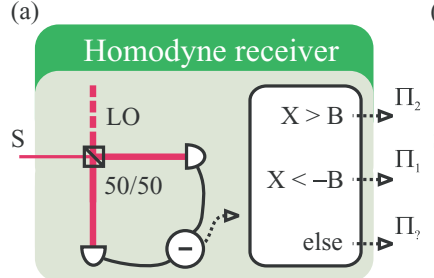

(c)

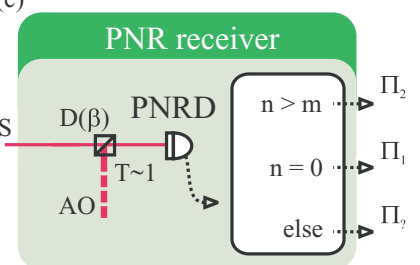

(b)

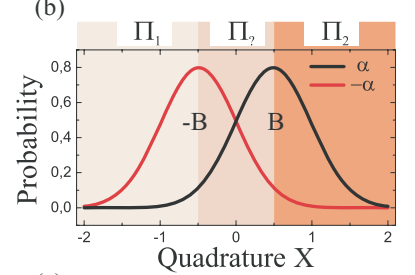

(e)

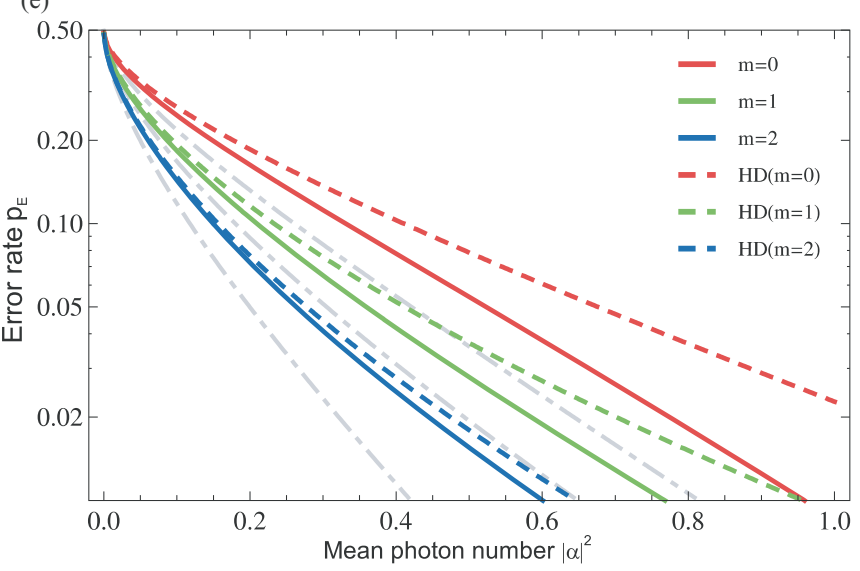

FIG. 1. (Color online) (a) Schematics of the homodyne receiver, showing that the signal (S) is interfered with a local oscillator (LO) on a 50:50 beam splitter. The photocurrents of two photodiodes are subtracted, resulting in a quadrature measurement along the encoding quadrature. (b) Marginal distribution of the two signal states. In the example, we assume a signal with $|\alpha|^{2}=0.24$. According to the measurement result the correct answer $(-, ?,+)$ is guessed. (c) Schematics of the photon number resolving (PNR) receiver. The signal $(\mathrm{S})$ is interfered with an auxiliary oscillator (AO) on a highly transmissive beam splitter. Finally, the signal is measured by a photon number resolving detector (PNRD). (d) Photon number distribution of two signal states. According to the measurement outcome of the PNRD the correct answer is guessed. In the example, we assume a signal with $|\alpha|^{2}=0.24$ and a displacement of $\beta=1$. Results for $n=1$ are considered inconclusive. (e) Comparison of PNR receivers with $m=1-3$ (solid lines) with homodyne receiver (dashed lines) at equal success rates. The PNR receiver outperforms the homodyne receiver for all signal amplitudes. The dot-dashed lines show the error rate of the optimal measurement.

where $\omega \in \mathbb{R}^{2 N}$ and $\hat{R}=\left[\hat{x}_{1}, \ldots, \hat{x}_{N}, \hat{p}_{1}, \ldots, \hat{p}_{N}\right]$. Here $\hat{x}_{i}$ and $\hat{p}_{i}$ are the quadrature operators of mode $i$. In particular, a Gaussian state is defined as the state whose characteristic function is represented by a Gaussian function [42],

$$
\chi_{\rho}(\omega)=\exp \left(-\frac{1}{4} \omega^{T} \Gamma \omega+i D^{T} \omega\right)
$$

where $\Gamma$ is a $2 N \times 2 N$ covariance matrix and $D$ is a $2 N$-vector corresponding to the displacement.
A similar formalism is applicable for the representation of POVMs. A single-mode POVM consisting of any Gaussian operation, Gaussian auxiliary states, and homodyne measurements can be described by a set of operators $\left\{\hat{\Pi}\left(d_{\mathcal{M}}\right)\right\}_{d_{\mathcal{M}}}$ whose characteristic function is [17]

$$
\begin{aligned}
\chi_{d}(\omega) & =\operatorname{Tr}\left[\hat{\Pi}(D) \exp \left(i \omega^{T} \hat{R}\right)\right] \\
& =\frac{1}{\pi} \exp \left(-\frac{1}{4} \omega^{T} \Gamma_{\mathcal{M}} \omega+i d_{\mathcal{M}}^{T} \omega\right),
\end{aligned}
$$

where $\Gamma_{\mathcal{M}}$ is a $2 \times 2$ covariance matrix and $d_{\mathcal{M}}=[u, v]^{T}$ represents the measurement outcome. A typical example of the measurement in this class is a heterodyne measurement described by $\{\hat{\Pi}(\beta)=|\beta\rangle\langle\beta| / \pi\}_{\beta \in \mathbb{C}}$ whose covariance matrix is calculated to be an identity matrix and $d_{\mathcal{M}}=$ $[\sqrt{2} \operatorname{Re} \beta, \sqrt{2} \operatorname{Im} \beta]^{T}$. Another example may be a homodyne measurement: A homodyne measurement with the phase $\varphi=0$ is a projection measurement onto an $X$ quadrature, and its covariance matrix is given by $\Gamma_{\mathcal{M}}=\operatorname{diag}\left[e^{-2 r}, e^{2 r}\right]$ with $r \rightarrow \infty$ and the element in the first row of $d_{\mathcal{M}}$ corresponding to the measurement outcome. Note that the POVM in Eq. (8) does not include conditional dynamics. In this formalism, the probability distribution of detecting a state $\hat{\rho}$ by a POVM $\hat{\Pi}\left(d_{\mathcal{M}}\right)$ is calculated as

$$
P\left(d_{\mathcal{M}}\right)=\operatorname{Tr}\left[\hat{\rho} \hat{\Pi}\left(d_{\mathcal{M}}\right)\right]=\frac{1}{2 \pi} \int d \omega \chi_{\rho}(\omega) \chi_{d}(-\omega) .
$$

More general characteristics of Gaussian state transformations in the formalism of characteristic function are described, for example, in $[40,42]$.

Let us construct the intermediate measurement via a Gaussian measurement described in Eq. (8), that is, without conditional dynamics, and classical postprocessing for a set of binary coherent states $\{|\alpha\rangle,|-\alpha\rangle\}$. When the measurement is "noise-free" (i.e., consisting of Gaussian unitary operations, pure Gaussian ancillary states, and ideal homodyne measurements) the covariance matrix is simply given by [17]

$\Gamma_{\mathcal{M}}=\left[\begin{array}{cc}\cosh 2 r-\sinh 2 r \cos \varphi & \sinh 2 r \sin \varphi \\ \sinh 2 r \sin \varphi & \cosh 2 r+\sinh 2 r \cos \varphi\end{array}\right]$,

where $r$ and $\varphi$ are real parameters. This noise-free restriction does not compromise generality since one can always construct a general Gaussian measurement by preparing a corresponding noise-free Gaussian measurement and discarding some of its measurement outcomes. We also note that such a noise-free Gaussian measurement corresponds to $\left\{\frac{1}{\pi}\left|\psi_{\zeta}(u, v)\right\rangle\left\langle\psi_{\zeta}(u, v)\right|\right\}_{(u, v)}$, where $\left|\psi_{\zeta}(u, v)\right\rangle=\hat{D}(u, v) \hat{S}(\zeta)|0\rangle, \hat{D}(u, v)=\exp [i(v \hat{x}-u \hat{p})]$ and $\hat{S}(\zeta)=\exp \left[\left(\zeta^{*} \hat{a}^{2}-\zeta \hat{a}^{\dagger 2}\right)\right]$ are a displacement and a squeezing operator, respectively, and $\zeta=r e^{i \varphi}$ is a complex squeezing parameter.

The characteristic functions of the coherent states $| \pm \alpha\rangle$ are given by $\chi_{ \pm}(\omega)=\exp \left(-\frac{1}{4} \omega^{T} I \omega+i d_{ \pm}^{T} \omega\right)$, where $I$ is the identity matrix and $d_{ \pm}=[ \pm \sqrt{2} \alpha, 0]^{T}$. The probability distribution of detecting $| \pm \alpha\rangle$ with such a POVM is thus 
calculated to be

$$
\begin{aligned}
P(u, v \mid \pm)= & \frac{1}{2 \pi} \int d \omega \chi_{ \pm}(\omega) \chi_{D}(-\omega) \\
= & \frac{1}{\pi \sqrt{\operatorname{det}\left(\Gamma_{\mathcal{M}}+I\right)}} \exp \left\{-\left[( \pm \sqrt{2} \alpha-u)^{2} a\right.\right. \\
& \left.\left.-2( \pm \sqrt{2} \alpha-u) v c+v^{2} b\right]\right\},
\end{aligned}
$$

where

$$
\frac{1}{\Gamma_{\mathcal{M}}+I}=\left[\begin{array}{ll}
a & c \\
c & b
\end{array}\right]
$$

and

$$
\begin{gathered}
a=\frac{1+\cosh 2 r+\sinh 2 r \cos \varphi}{2(\cosh 2 r+1)}, \\
b=\frac{1+\cosh 2 r-\sinh 2 r \cos \varphi}{2(\cosh 2 r+1)}, \\
c=\frac{-\sin 2 r \sin \varphi}{2(\cosh 2 r+1)} .
\end{gathered}
$$

We first show that the optimal measurement in this class is homodyne detection. Let us denote the likelihood ratio of two signals as

$$
\Lambda_{1}=\frac{p_{1} P(u, v \mid-\alpha)}{p_{2} P(u, v \mid \alpha)}=\frac{p_{1}}{p_{2}} \exp [-4 \sqrt{2} \alpha(a u+c v)],
$$

and $\Lambda_{2}=\Lambda_{1}^{-1}$. According to the Bayesian strategy [4], an optimal signal decision for the fixed measurement is to guess $|-\alpha\rangle$ for $\Lambda_{1} \geqslant \Lambda_{B},|\alpha\rangle$ for $\Lambda_{2} \geqslant \Lambda_{B}$, and the inconclusive result otherwise, where $\Lambda_{B}$ is the threshold. The probabilities of having successive, erroneous, and inconclusive results for each signal are then given by

$$
\begin{gathered}
p_{s}^{( \pm)}=\frac{1}{2} \operatorname{erfc}\left(\sqrt{2 a} \alpha-\frac{\ln \Lambda_{B} \pm \ln \left(p_{1} / p_{2}\right)}{4 \sqrt{2} \alpha}\right), \\
p_{e}^{( \pm)}=\frac{1}{2} \operatorname{erfc}\left(\sqrt{2 a} \alpha+\frac{\ln \Lambda_{B} \mp \ln \left(p_{1} / p_{2}\right)}{4 \sqrt{2} \alpha}\right), \\
p_{i}^{( \pm)}=p_{s}^{( \pm)}-p_{e}^{( \pm)} .
\end{gathered}
$$

The average error and inconclusive probabilities are given by $p_{E}=\left(p_{1} p_{e}^{(-)}+p_{2} p_{e}^{(+)}\right) /\left(1-p_{\text {inc }}\right)$ and $p_{\text {inc }}=p_{1} p_{i}^{(-)}+p_{2} p_{i}^{(+)}$, respectively. We find that, for a given $\Lambda_{B}$, these two probabilities are simultaneously minimized for $\varphi=0$ and $r \rightarrow \infty$, that is, an ideal homodyne measurement with phase $\varphi=0$. This implies that the optimal measurement with only Gaussian operations is the homodyne detector with a fixed phase of $\varphi=0$.

Furthermore, in the following we prove that any conditional operation will not improve the discrimination task. To prove this, we consider two different Gaussian operators. The first operation is a partial measurement of the signal which in general outputs a measurement outcome (classical number) and a conditioned output state. For an input of $p_{1}|-\alpha\rangle\langle-\alpha|+$ $p_{2}|\alpha\rangle\langle\alpha|$, the conditioned output is given as

$$
\hat{\rho}_{\text {out }}=p_{1}^{\prime}\left(d_{\mathcal{M}}\right) \hat{\rho}_{-}+p_{2}^{\prime}\left(d_{\mathcal{M}}\right) \hat{\rho}_{+},
$$

where $\hat{\rho}_{ \pm}$are general multimode states that preserve Gaussian statistics with the joint covariance matrix $\Gamma_{\text {out }}$ and the displacement $\pm D$. Here $d_{\mathcal{M}}$ denotes the measurement outcome and thus only the a posteriori probabilities in Eq. (20) depend on $d_{\mathcal{M}}$. Moreover, it was shown that $\hat{\rho}_{\text {out }}$ can always be transformed into another mixture of coherent states [17],

$$
\hat{\rho}_{\text {out }} \rightarrow \hat{\rho}_{\alpha^{\prime}} \otimes \hat{\rho}_{\text {aux }},
$$

where $\hat{\rho}_{\text {aux }}$ is some Gaussian state and

$$
\hat{\rho}_{\alpha^{\prime}}=p_{1}^{\prime}\left(d_{\mathcal{M}}\right)\left|-\alpha^{\prime}\right\rangle\left\langle-\alpha^{\prime}\left|+p_{2}^{\prime}\left(d_{\mathcal{M}}\right)\right| \alpha^{\prime}\right\rangle\left\langle\alpha^{\prime}\right|,
$$

with real and positive $\alpha^{\prime}$. Such an additional Gaussian operation can be deterministic and independent of the partial measurement outcome $d_{\mathcal{M}}$. Since only the a posteriori probabilities depend on $d_{\mathcal{M}}$, the optimal second operation is independent of $d_{\mathcal{M}}$ and given by a fixed homodyne measurement $(\varphi=0)$ as already shown. We therefore conclude that any conditional dynamics is not useful in the two-step measurement scenario. An extension of the above conclusion to the multistep measurement scenario is straightforward, which proves the optimality of the homodyne detector within all possible Gaussian operations and conditional dynamics.

\section{PHOTON NUMBER RESOLVING RECEIVER}

Quadrature measurements (measurements of the light's field amplitude) and photon counting measurements (measurements of the excitation of a light field) are fundamentally different. Therefore, it is of interest to investigate also a receiver based on the latter technique. In Ref. [25] we proposed to use a photon number resolving receiver for the discrimination of two coherent states. It consists of two stages: a displacement operation $D(\beta)$ and a photon number resolving detector (PNRD), and it is sketched in Fig. 1(c).

The postselection process of the PNR receiver is similar to that of the homodyne detector: If the measurement outcome of the PNR detector is $n=0$, we guess $|-\alpha\rangle$, if it is $n>m$ (where $m$ is the threshold parameter), we guess $|\alpha\rangle$, and otherwise the measurement is inconclusive. This can be described by the projector $\hat{\Pi}_{?}=\sum_{n=1}^{m}|n\rangle\langle n|$ for $m>0$. Conclusive results are described by $\hat{\Pi}_{1}=|0\rangle\langle 0|$ and $\hat{\Pi}_{2}=$ $\hat{I}-\hat{\Pi}_{1}-\hat{\Pi}_{\text {? }}$, where $\hat{\Pi}_{1}$ identifies $|-\alpha\rangle$ and $\hat{\Pi}_{2}$ identifies $|\alpha\rangle$. An example for the photon number distributions of two displaced coherent states is shown in Fig. 1(d). The error rate is then given by

$$
p_{E, \mathrm{PNR}}=\frac{\left(1-\frac{\Gamma\left(m+1,(\alpha-\beta)^{2}\right)}{\Gamma(m+1)}+e^{-(\alpha+\beta)^{2}}\right)}{2\left(1-p_{D, \mathrm{inc}}\right)},
$$

where the Euler gamma function $\Gamma(z)$ and the incomplete gamma function $\Gamma(a, z)$ are defined as $\Gamma(z)=\int_{0}^{\infty} t^{z-1} e^{-t} d t$ and $\Gamma(a, z)=\int_{z}^{\infty} t^{a-1} e^{-t} d t$. The probability of inconclusive results is given by

$$
\begin{aligned}
p_{\text {inc }, \mathrm{PNR}}= & \frac{\Gamma\left(m+1,(\alpha-\beta)^{2}\right)+\Gamma\left(m+1,(\alpha+\beta)^{2}\right)}{2 \Gamma(m+1)} \\
& -\frac{1}{2} e^{-(\alpha-\beta)^{2}}-\frac{1}{2} e^{-(\alpha+\beta)^{2}} .
\end{aligned}
$$

The displacement in the receiver can be chosen such that one of two input states is displaced to the vacuum state $(\beta=\alpha)$ as suggested by Kennedy [11]. However, to minimize the error rate of the receiver the displacement must be optimized, that 
is, $d p_{E, \mathrm{PNR}} / d \beta=0$. A detailed discussion of this receiver can be found in Ref. [25].

We compare the PNR receiver with homodyne receivers with different postselection thresholds. In this comparison, we choose the postselection parameter $B$ such that the rates of inconclusive results are equal for both strategies, that is, $p_{\text {inc }, \mathrm{HD}}=p_{\text {inc,PNR}}$. The error probability for the receivers with $m=0-2$ and the corresponding homodyne receivers are plotted against the mean photon number of the signal in Fig. 1(e). We find that the performance of the PNR receiver (solid lines) surpasses the performance of the homodyne receiver (dashed lines) for all signal amplitudes. The optimal discrimination strategy is shown by the dot-dashed, gray curve.

\section{EXPERIMENTAL RESULTS}

In the following section, the receiver schemes are demonstrated with the experimental setup shown in Fig. 2. It consists of a preparation stage and two different receiver stages; the PNR receiver and a homodyne receiver. Our source is a grating stabilized cw diode laser at $810 \mathrm{~nm}$ (Toptica DL100). After passing a fiber mode cleaner, the linearly polarized beam is split asymmetrically into two parts to serve as a local oscillator of the homodyne receiver (LO) and an auxiliary oscillator for state preparation and displacement in the PNR receiver scheme. The signal state $(\mathrm{S})$ is generated in a polarization mode orthogonal to the auxiliary mode using an electro-optical modulator (EOM): The field amplitude of the auxiliary mode

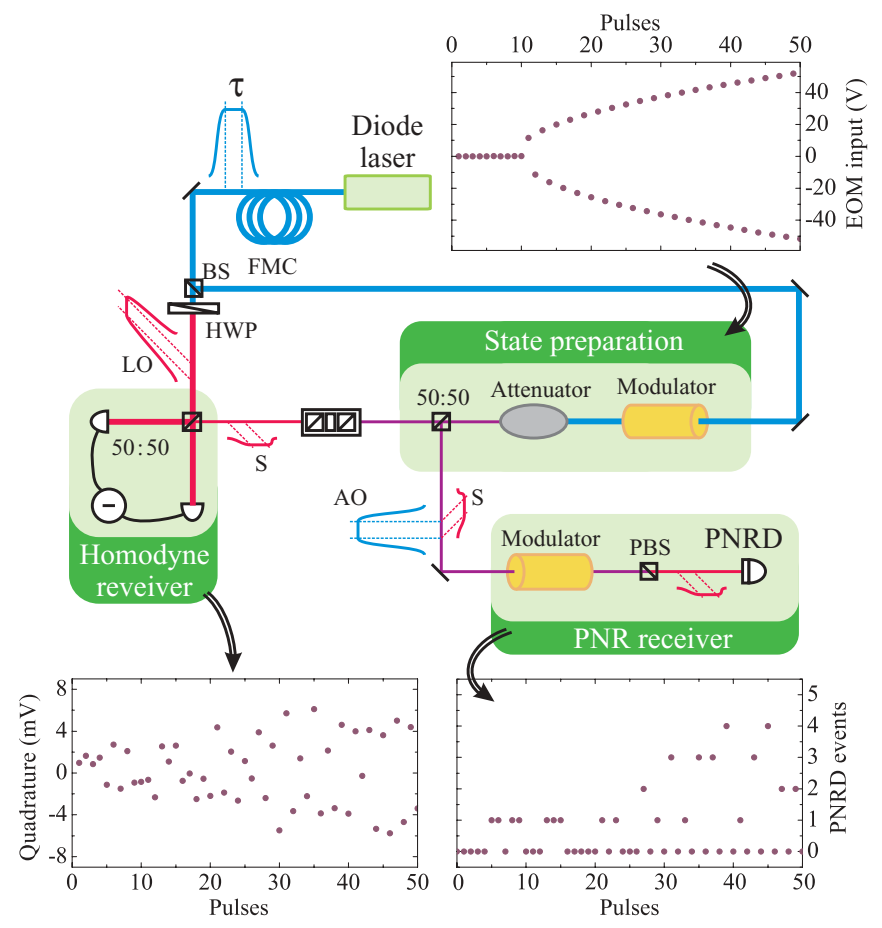

FIG. 2. (Color online) Simplified scheme of the experiment, where the abbreviated components are a fiber mode cleaner (FMC), beam splitters (BSs, 50:50), a polarizing beam splitter (PBS), a half-wave plate (HWP), and a photon number resolving detector (PNRD). The graphs show modulation and the corresponding recorded quadrature measurements and detection events of the PNRD. is coherently transferred into the signal polarization and the excitation is controlled by the input voltage of the modulator. Note that the auxiliary oscillator remains in the polarization mode orthogonal to the signal mode, thus propagating along with the signal. After the signal was split on a 50:50 beam splitter, two identical signal states (either $|\alpha\rangle^{\otimes 2}$ or $|-\alpha\rangle^{\otimes 2}$ ) are produced and subsequently directed to the two detection schemes.

The signal states are generated in time windows of $\tau=$ $800 \mathrm{~ns}$ with a repetition rate of $100 \mathrm{kHz}$. This was done by applying a constant voltage across the modulator during the measurement time. The birefringence induced by the EOM's input voltage causes a variable coupling between the $\mathrm{S}$ and $\mathrm{AO}$ mode similarly to a variable beam splitter. We can therefore tune the signal amplitude continuously.

This modulation scheme is in contrast to the commonly used sideband modulation approach in experiments with homodyne detectors, where a rf modulation is applied to the modulator to create a pair of frequency sideband modes that defines the quantum state. Since an avalanche photodiode (APD) is not capable of selecting a specific pair of sideband modes, such a sideband approach cannot be used when the homodyne detector is used in conjunction with an APD. The quantum states are therefore defined as a pulse in the temporal frame of the local oscillator. The exact pulses measured by the two detectors are not identical as they have different frequency responses. The effect of the detector response is described after the detailed description of the detection schemes.

We carefully characterize the prepared input signal and verify that the excess noise added to the quadrature by the signal preparation is only $5 \times 10^{-3}$ shot noise units (see Ref. [21]). This purity is achieved by attenuation of the laser (the carrier) down to the single-photon level, thereby minimizing the thermal fluctuations at low frequencies prevailing in normal diode laser operation.

At the homodyne receiver the signal interferes with the local oscillator, the two resulting outputs are detected, and a difference current is produced. This yields an integrated quadrature value for each signal pulse. The detected signal of the homodyne detector is filtered with a seven-pole Chebyshev low-pass filter from dc to $10 \mathrm{MHz}$, and subsequently the signal is sampled at the rate $20 \mathrm{MS} / \mathrm{s}$. For a single pulse, the number of samples produced was therefore 16. These data were then averaged, thus resulting in a single quadrature measurement for an $800 \mathrm{~ns}$ pulse. The technical noise at low frequencies was removed by correcting for the baseline shift occurring between consecutive signal states. The phase of the signal relative to the LO is estimated by sending a number of bright calibration pulses along with the signal pulses. Subsequently, the measurements are accepted or discarded according to the estimated phase, that is, they are accepted only if the measurement was performed along the signal encoding quadrature. This substitutes for a technically demanding phase-locking method. A drawback is the increased measurement time. The overall quantum efficiency of the homodyne receiver amounts to $\eta_{\text {hom }}=85.8 \%$; the interference contrast to the local oscillator is $(96.6 \pm 0.1) \%$ and the $p-i-n$ diode quantum efficiency is $(92 \pm 3) \%$. The electronic noise level is more than $23 \mathrm{~dB}$ below the shot noise level. 
The PNR receiver is composed of a displacement operation and a fiber-coupled avalanche photodiode operating in an actively gated mode to circumvent a dark count event and thus a dead time at the time the pulse is arriving. During the measurement time, the APD works as a primitive photon number resolving detector, as used in [43]. The quantum states are subsequently categorized according to the corresponding result of the photon number measurement, thus implementing the POVMs $\hat{\Pi}_{1}=|0\rangle\left\langle 0\left|, \hat{\Pi}_{?}=\sum_{n=1}^{m}\right| n\right\rangle\langle n|$, and $\hat{\Pi}_{2}=\hat{I}-$ $\hat{\Pi}_{1}-\hat{\Pi}_{\text {? }}$. In contrast to the displacement operation depicted in Fig. 1(b) where two spatially separated modes interfere on a beam splitter, in our setup the two modes (the auxiliary and the signal modes) are in the same spatial mode but have different polarization modes [Fig. 1(c)]. The interference (and thus the displacement) is therefore controlled by a modulator and a polarizing beam splitter. This method facilitates the displacement operation and yields a very high interference contrast of $99.6 \%$. A high extinction ratio is of great importance as the mismatched part of the auxiliary might impinge onto the APD and cause false detection events. Such false counts can be detrimental to the discrimination task for receivers with low error rate and especially if the signal amplitude is relatively large.

The detection efficiency of the scheme is estimated as $\eta_{\text {on } / \text { off }}=55 \%$, including the transmission coefficient of the modulator, the polarization optics, and the fiber of $89.1 \%$ as well as the quantum efficiency of the APD of $(63 \pm 3) \%$. The last efficiency was estimated by the APD click statistic for an input coherent state that was calibrated by the homodyne receiver. An optical isolator is used between the two detection schemes to prevent backscattering of the LO to the APD.

Because the two detectors have different responses, they do not measure the exact same temporal mode. However, in the following we show that the two different temporal modes are nearly identical, possessing an overlap of about $95 \%$. In the experiment, we probe the optical mode $\hat{a}\left(t_{m}\right)=$ $(1 / T) \int_{t_{m}-T / 2}^{t_{m}+T / 2} \hat{a}(t) d t$. The APD is a broadband detector, and by neglecting the electronic jitter noise and the dead time, the APD detects the following mean photon number during the measurement time $T$ :

$$
\hat{n}\left(t_{m}\right)=\frac{1}{T} \int_{t_{m}-T / 2}^{t_{m}+T / 2} \hat{a}^{\dagger}(t) \hat{a}(t) d t .
$$

On the other hand, the homodyne detector has a finite detector bandwidth, which means that the quadrature measurement $\hat{X}_{\text {sa }}$ at time $t$ depends on the detector response $G(\tau)$. A single sample is thus described by [44]

$$
\hat{X}_{\mathrm{sa}}(t)=\int d \tau G(\tau) \hat{X}(t-\tau),
$$

where $G(\tau)$ is determined by the detector's frequency response $G(\omega)$ and accounts for the mean of $\hat{X}_{\mathrm{sa}}$ over the measurement time $T$. The time-averaged measurement $\hat{X}_{\text {av }}$ at the time $t_{m}$ can then be written as

$$
\begin{aligned}
\hat{X}_{\mathrm{av}}\left(t_{m}\right) & =\frac{1}{T} \int_{t_{m}-T / 2}^{t_{m}+T / 2} d t \int d \tau G(\tau) \hat{X}(t-\tau) \\
& =\frac{1}{T} \iint d t d \tau \operatorname{rect}\left(\frac{t-t_{m}}{T}\right) G(t-\tau) \hat{X}(\tau)
\end{aligned}
$$

$$
\begin{aligned}
& =\frac{1}{T} \int d \tau \hat{X}(\tau) \int d t \operatorname{rect}\left(\frac{t-t_{m}}{T}\right) G(t-\tau) \\
& \widehat{=} \int d \tau G_{\text {eff }}\left(t_{m}-\tau\right) \hat{X}(\tau)
\end{aligned}
$$

where the measurement is described by the effective response function $G_{\text {eff }}$. We compare this function to the ideal mode $\hat{X}_{\text {ideal }}\left(t_{m}\right)=(1 / T) \int_{t_{m}-T / 2}^{t_{m}+T / 2} d t \hat{X}(t)$ as illustrated in Fig. 4 . We estimate the mode overlap of the ideal and the effective modes with the normalized cross-correlation function $g_{12}=$ $\left\langle\hat{X}_{\text {av }} \hat{X}_{\text {ideal }}\right\rangle / \sqrt{\left|\hat{X}_{\text {av }}\right|^{2}\left|\hat{X}_{\text {ideal }}\right|^{2}}$ (which is related to the visibility for the interference of two partially coherent waves [45]). For our detector the cross-correlation is $95.5 \%$, and thus the similarity between the temporal mode experienced by the APD and the one experienced by the homodyne detector is about $95 \%$.

We proceed by describing the procedure for the discrimination task. A PC controls the preparation of the states and the displacement in the PNR receiver by modulating two electro-optical modulators. Simultaneously it acquires the homodyne and APD detection outcomes during the pulse sequence. An example of such a sequence is shown in Fig. 2, where we show the voltages applied to the amplifiers, the quadrature values, and the recorded number of counts per measurement time. The outcomes of the homodyne receiver within the interval $[-B, B]$ are considered as inconclusive results. If the value is outside the interval and positive we guess $|\alpha\rangle$, and if the value is outside the interval and negative we guess $|-\alpha\rangle$. For the outcomes of the PNR receiver, we use the hypothesis that, if the outcome is larger than $m$, we guess $|\alpha\rangle$, if it is zero, we guess $|-\alpha\rangle$, and otherwise it is an inconclusive result. The error probability is therefore found by addition of all the false detections related to the number of pulses that were accepted. The acceptance probability is the ratio of pulses that were accepted to the total number of pulses.

The theoretical predictions for the acceptance probability $1-p_{\text {inc,HD }}$ and the error probability $p_{E, \mathrm{HD}}$ are shown in Figs. 3(a) and 3(b), respectively. For increasing signal amplitude $|\alpha|^{2}$ the error probability $p_{E \text {,HD }}$ drops, and an increase of the postselection threshold $B$ leads to a decreasing error probability at the expense of an increase in the probability for inconclusive results. An advantage of the homodyne receiver is the smooth dependence between postselection threshold and error rate. This allows one to chose exactly the error rate desired for a specific application. For example, in quantum key distribution the amount of mutual information between sender, adversary, and receiver can be easily adjusted through the postselection threshold [26]. The receiver's performance is completely characterized by the error and acceptance rates. In Fig. 3, we introduced a red dashed line, where the condition $p_{\text {inc, HD }}=p_{\text {inc, USD }}$ is met, with $p_{\text {inc, USD }}$ the probability of inconclusive results in a perfect USD measurement. This means that an error-free but probabilistic discrimination is in principle possible above this curve.

The experimental results for the acceptance and the error probability of the homodyne receiver are shown in Figs. 3(b) and 3(d), respectively. The contour plots are generated from signals with 21 different amplitudes (with linearly increasing mean photon number) and calculated for 41 postselection thresholds. We find very good agreement of theory and 
(a) Acceptance probability $1-p_{\text {inc,HD }}$ (theor.)

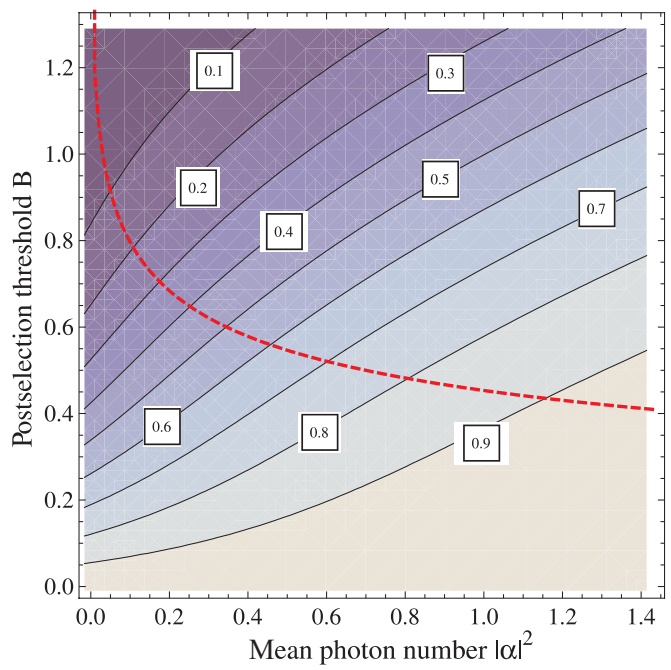

(b) Error probability $\log _{10}\left(p_{\mathrm{E}, \mathrm{HD}}\right)$ (theor.)

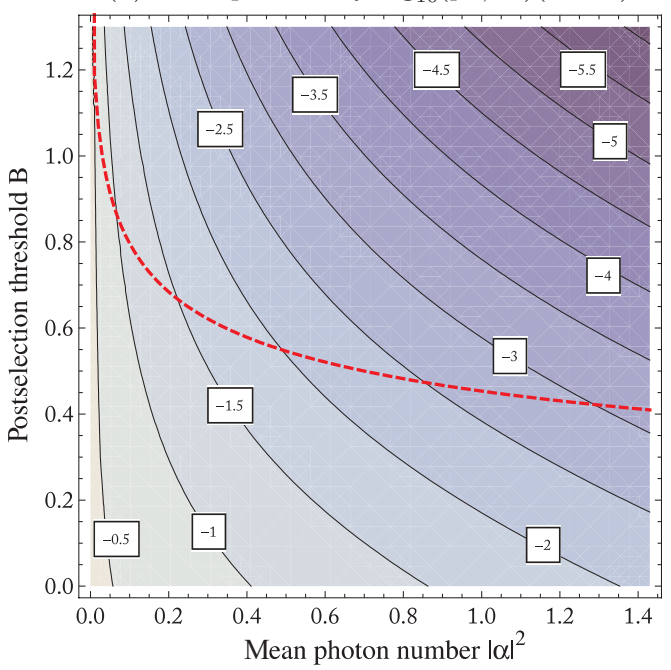

(c) Acceptance probability $1-p_{\text {inc,HD }}$ (expt.)

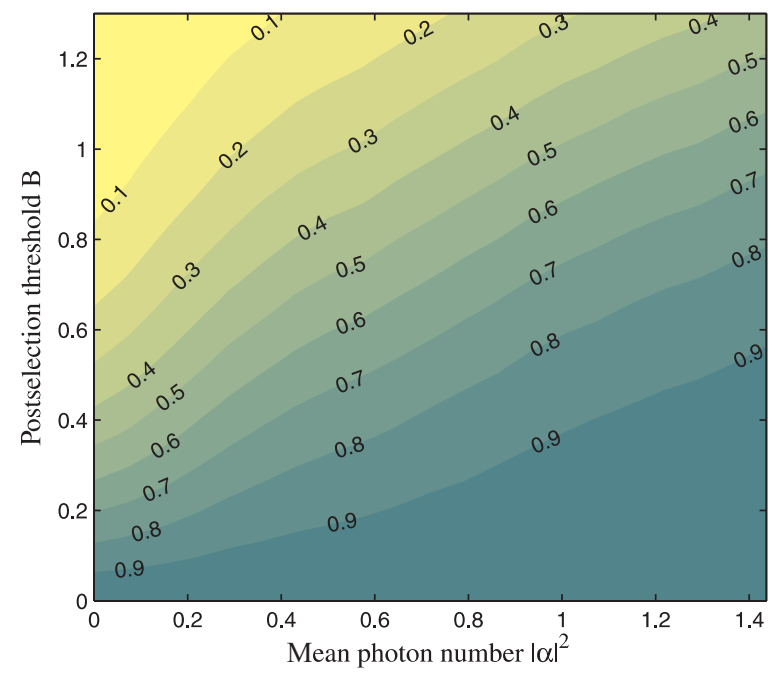

(d) Error probability $\log _{10}\left(p_{\mathrm{E}, \mathrm{HD}}\right)$ (expt.)

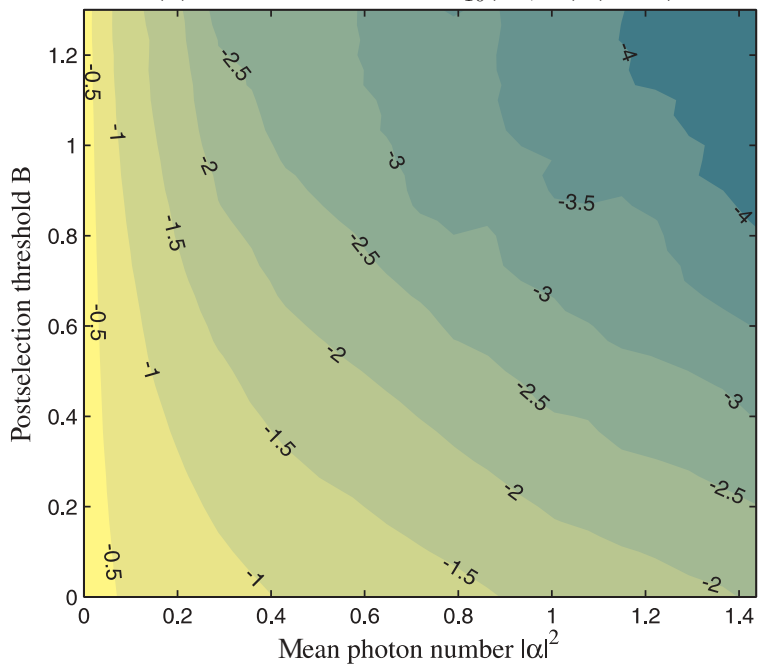

FIG. 3. (Color online) (a) Dependence of the acceptance probability for ideal homodyne detection on the signal's mean photon number $|\alpha|^{2}$ and the postselection threshold $B$. The dashed line shows the postselection threshold $B$ for which the homodyne detection and USD have equal acceptance probability. (b) The error probabilities of ideal homodyne detection shown in a logarithmic contour plot. The dashed line shows the error rate of homodyne detection for success rates equal to USD. (c) Experimentally measured acceptance probability. (d) Experimentally measured error probabilities. In (c) and (d), we corrected for the quantum efficiency of the receiver.

experiment with only minor deviations for very low error probabilities.

The PNR receiver is demonstrated for $m=0,1$, and 2 . In Fig. 5(a), the dependence of the error probability on the displacement $\beta$ for a fixed signal amplitude is illustrated. We

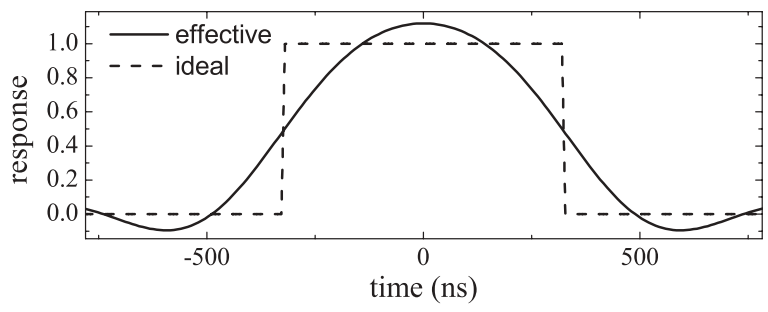

FIG. 4. Comparison between the effective and ideal response functions of the homodyne receiver. find that for any $m$ the displacement can be optimized such that the experimentally measured error rates reach a minimum. The optimal displacement is higher for higher $m$, and the minimum error rate after this optimization of the displacement is lower for increasing $m$. When compared to the theoretical predictions, the experimental data fit well in the region of the minima, while the experimental imperfections dominate in the region of smaller displacement. We also observed this for $m>2$.

We marked four data points in the plot. From left to right, they represent the error rates associated with the Kennedy receiver (black squares) (an early receiver for minimum error discrimination [11] without optimized displacement, i.e., $\beta=\alpha$ ), the optimized displacement receiver with $m=0$ (red circles), and the PNR receivers with $m=1$ and 2 (green triangles and blue diamonds). The error rates for varying amplitudes are plotted in Fig. 5(b). We find a maximal 
(a)
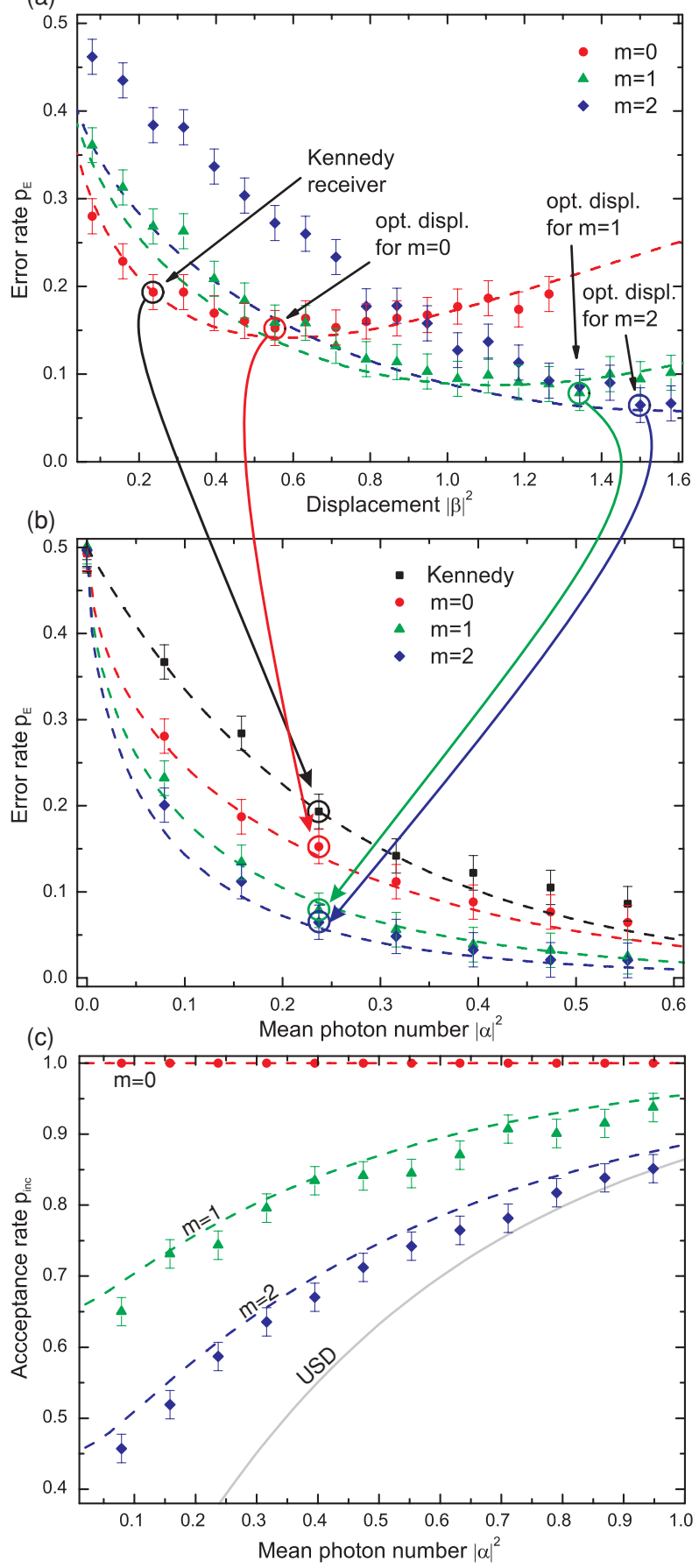

FIG. 5. (Color online) (a) Experimental data showing the effect of the displacement $\beta$ on the error rate $p_{E \text {,PNR }}$ for a given signal amplitude $|\alpha|^{2}=0.24$ (corrected for quantum efficiency) and different numbers of dropped results $m$. The theoretically predicted error probabilities for the receiver without imperfections are shown with dashed lines. The error rates at the optimal displacement $\beta_{\text {opt }}$ for different receivers are marked. (b) Error rates for varying $m$ and optimized $\beta_{\text {opt }}$. The error bars reflect the standard deviations of repeated measurements, which are larger than the statistical errors. The experimental data are compared to ideal receivers (dashed lines). (c) Experimental data for acceptance rates (points) and theoretical predictions (dashed lines). Below the curve for an optimal USD device (solid gray), states can be discriminated without error in principle. We corrected for the quantum efficiencies of the receiver.
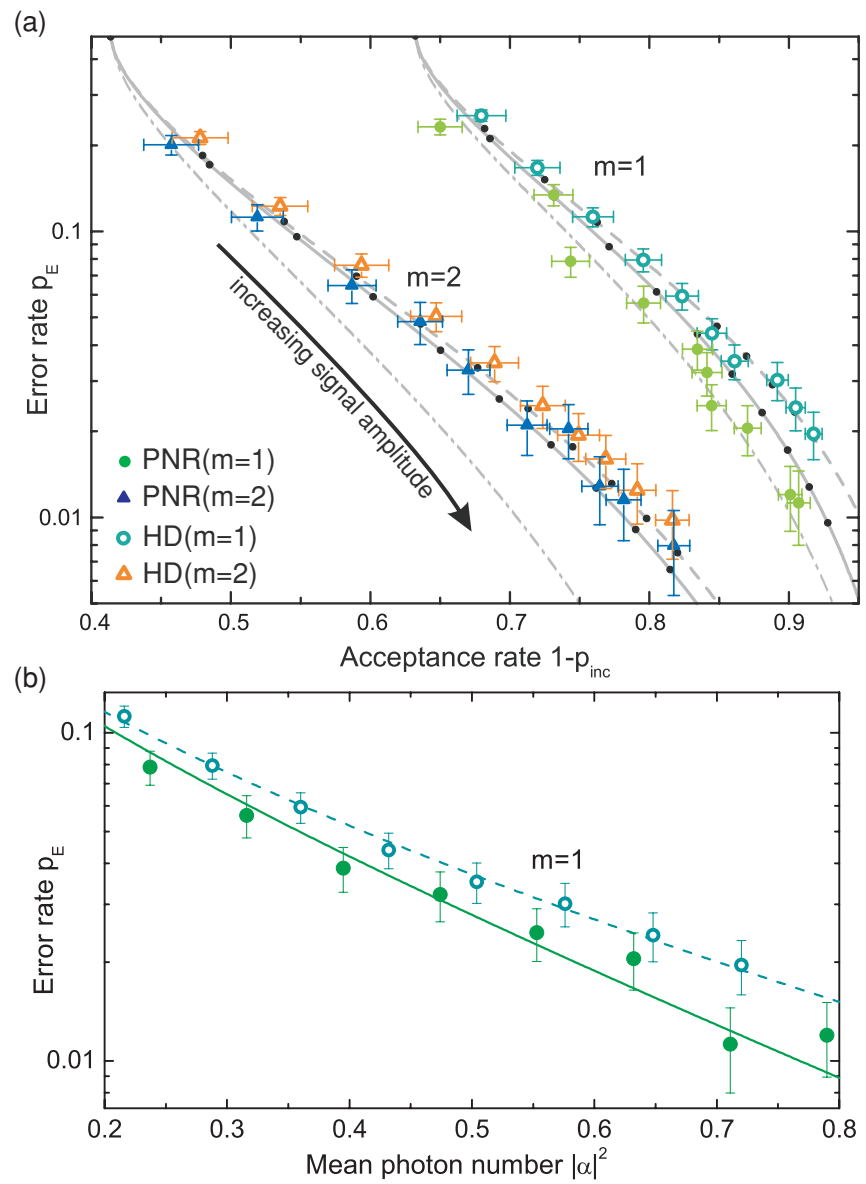

FIG. 6. (Color online) (a) Comparison of experimental error rates and acceptance rates for the two receiver schemes. For this comparison the success rate of both schemes is fixed to the one that is theoretically reached by the PNR receiver. Experimental data are shown for PNR receivers with $m=1$ and 2 (filled circles and triangles) and the homodyne receiver (open circles and triangles). Additionally, theoretical predictions for the homodyne receiver (dashed line), the PNR receiver (solid line), and the optimal intermediate measurement (dot-dashed lines) are shown. The mean photon number is varied for the different receivers. The PNR receiver again outperforms the homodyne receiver, and we find a relatively good agreement of experimental data and theoretical predictions. (b) Error rate for various signal amplitudes. The PNR receiver surpasses the homodyne receiver. Statistical error bars show standard deviation of the random process. We corrected for the quantum efficiencies of the receivers.

reduction of the error rate by a factor of 3.5 in going from $m=0$ (deterministic scheme) to $m=2$ (probabilistic scheme) at the signal amplitude $|\alpha|^{2}=0.47$. The corresponding penalty on the acceptance rates and a comparison with the theoretical predictions for the acceptance probability are shown in Fig. 5(c).

The two detection schemes are compared to each other in Fig. 6. We find that both receivers show the expected behavior. In particular for $m=1$, it is obvious that the PNR receiver outperforms the homodyne receiver for several data points.

In the following, we discuss the limitations of the different schemes. The quantum efficiency of homodyne detection is partly limited by the $p-i-n$ diode efficiency and partly by the mode-matching efficiency at the homodyne's beam splitter. 
For specially made $p-i-n$ diodes, the efficiency can reach nearly $100 \%$, and beam splitter mode-matching efficiencies beyond $99 \%$ have been reported. The efficiency of the PNR is mainly limited by the quality of the displacement operation and the efficiency of the avalanche photodiode. For higher values of $m$, we also find that the probability of false detection events becomes important [see $m=2$ in Fig. 6(a)]. We used a commercially available APD, but the development of photon number resolving detectors with very high quantum efficiency is rapidly progressing (see [46] for a detailed list).

\section{APPLICATION OF PNR RECEIVERS IN QKD}

In the following, we show that PNR detection can effectively replace homodyne detection in certain standard quantum information protocols such as QKD. To demonstrate its advantage, we take the standard binary continuous-variable (CV) QKD protocol [47] and replace the homodyne detection part with the PNR receiver described above. The main steps of the protocol are the following.

1. The sender (Alice) randomly chooses a coherent state from a binary alphabet $(| \pm \alpha\rangle)$ and sends it to the receiver (Bob).

2. Bob performs an optimal displacement on the attenuated state $| \pm \sqrt{\eta} \alpha\rangle$ by the value $\beta$ and measures the state $|\beta \pm \sqrt{\eta} \alpha\rangle$ with a PNR detector, thereby obtaining a result $n$. In the case of $n=0$, he associates the result with the state $|\beta-\sqrt{\eta} \alpha\rangle$, and in the case of $n>m$, with the state $|\beta+\sqrt{\eta} \alpha\rangle$. In the remaining cases $0<n \leqslant m$, he gets an inconclusive result with probability $p_{\text {inc }}$ [see Eq. (24)] and thus omits the transmitted states associated with these data.

3. After collecting a sufficiently large set of data, Alice and Bob perform the error correction and privacy amplification procedures.

For the purpose of comparing PNR and double-homodyne detection, we perform a security analysis of the protocol with direct reconciliation in the case of a noiseless quantum channel and assuming the optimal collective attack by Eve. Under these assumptions, the security analysis is similar to the one developed in $[47,48]$. The amount of secret information per single transmission (i.e., the secret key rate) is equal to the difference between Bob's and Eve's information.

During error reconciliation with direct communication (Alice sends classical information to Bob), Eve has to guess the quantum states that were transmitted by Alice. Thus her information does not depend on Bob's measurement strategy and is essentially the same as for homodyne detection [47]:

$$
I_{E}=-\left[c \log _{2} c+(1-c) \log _{2}(1-c)\right],
$$

where $c=\left(1+e^{-2(1-\eta) \alpha^{2}}\right) / 2$.

Bob's information reflects the amount of correlations in the accepted part of all measurements (i.e., when $n=0$ or $n>m$ ) and can be calculated as Shannon information:

$$
I_{B}=1-H\left(p_{\mathrm{err}}\right),
$$

where $p_{\text {err }}$ is given by Eq. (23).

The secret key rate is equal to the average information advantage of Bob over Eve:

$$
G=\left(1-p_{\text {inc }}\right)\left(I_{B}-I_{E}\right) .
$$

The first term $\left(1-p_{\text {inc }}\right)$ takes into account only conclusive results after Bob's measurement, and the second term $\left(I_{B}-\right.$ $I_{E}$ ) corresponds to the information contribution of the accepted transmissions.
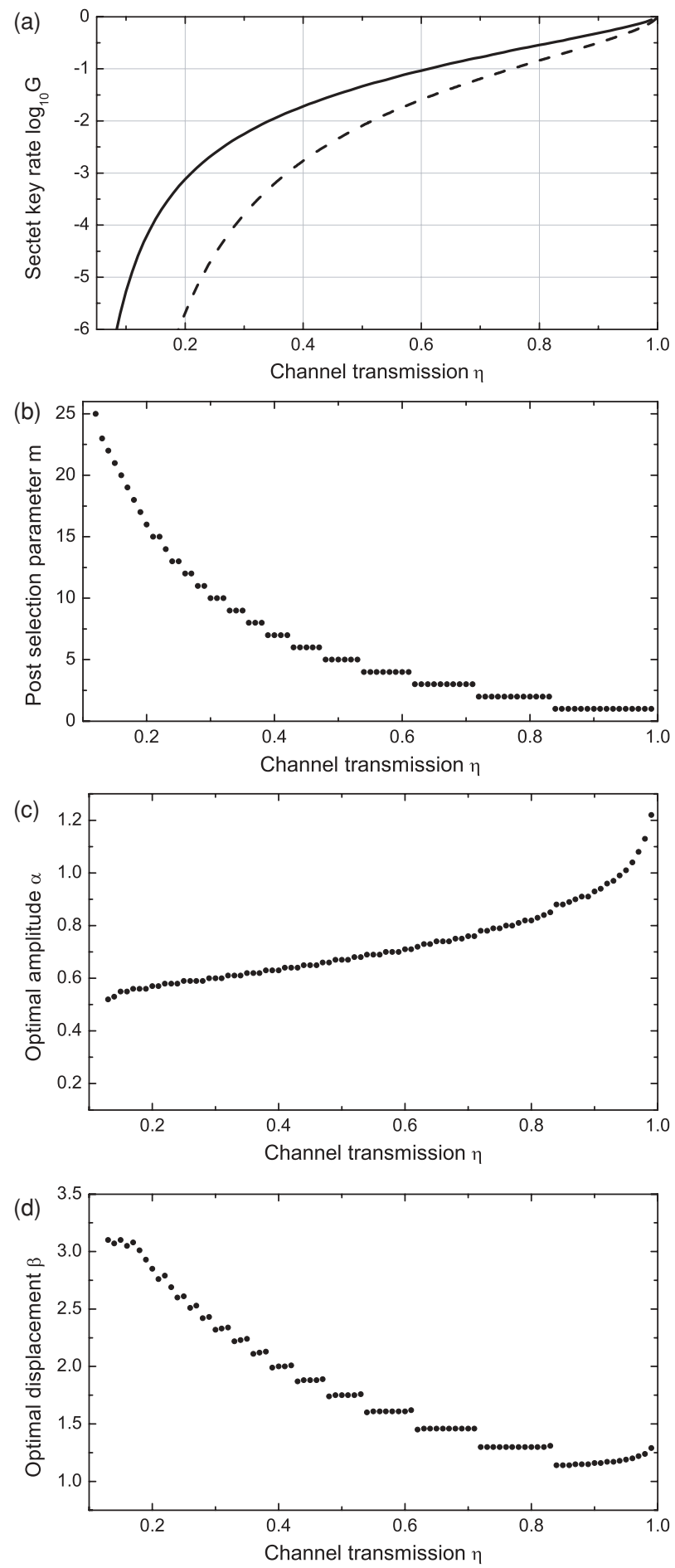

FIG. 7. (a) Key rate $G$ on logarithmic scale $\left(\log _{10} G\right)$ as a function of the channel transmittance $\eta$. The solid curve corresponds to the optimized PNR receiver. The dashed curve shows the secret key rate for the decoding strategy based on optimized homodyne detection $[47,48]$. The other figures show the optimized (b) threshold number $m$, (c) signal amplitude $\alpha$, and (d) displacement $\beta$ as functions of the channel transmittance. 
The key rate (30) depends on four variable parameters: the signal's amplitude $\alpha$, the displacement amplitude $\beta$, the threshold value $m$, and the channel transmittance $\eta$. As a standard figure of merit for characterization of the QKD protocol quality, we show the key rate $G$ as a function of the channel transmittance $\eta$, assuming optimization of all other parameters $(\alpha, \beta$, and $m$ ). The exact optimization of these parameters is performed numerically in order to maximize the key rate. If the optimization procedure is different (e.g., minimization of the error rate or minimization of the probability of an inconclusive result), then the parameters obtained are also different and do not lead to the maximum key rate.

After optimization of all parameters, we found that the optimal amplitude $\alpha$ is similar to that of the double-homodyne detection scheme [48] and is about $\alpha \simeq 0.5-1$, the optimal displacement parameter is about $\beta \simeq 1-3$, and the optimal threshold value $m$ ranges from 1 to 30 . The exact results are shown in Fig. 7. We can see that the CV QKD protocol based on the PNR receiver is better than the one based on a homodyne receiver, especially in the case of high channel attenuation.

\section{CONCLUSION}

In this paper, we have thoroughly investigated two different probabilistic receivers of binary-encoded optical coherent states; the homodyne detector and the displacement-controlled photon number resolving detector. These receivers yield inconclusive as well as error-affected results, and we have carefully conducted a detailed study of the relation between these two outcomes. Furthermore, we found, theoretically, that the homodyne detector is the optimal Gaussian receiver for minimizing the errors for a fixed probability of inconclusive results. Experimentally, we have implemented both receivers and through comparison we found that the performance of the displacement-controlled PNR receiver is better than that of the homodyne receiver.
The PNR receiver is thus a promising alternative to the commonly used homodyne receiver. We find several advantages of this scheme. For example if the phase reference is sent along with the signal (most CV QKD scenarios), the power of the displacement beam is normally much lower than the power of the local oscillator beam required for homodyne detection, and thus less power is injected into the communication channel (e.g., an optical fiber). This has the obvious benefit of lowering the power consumption in the fiber, but it also lowers the risk of scattering of auxiliary photons into the signal state, as this scattering mechanism is proportional to the power. We also note that, instead of performing the displacement operation at the receiving station, it can already be implemented at the sending station. This would completely remove the necessity for a phase reference. Finally, we note that the quadrature measurement can also be performed using a displacement operation followed by a single intensity detector, similar to the setup of the PNR detector. However, in that case, the displacement must be macroscopic so that the quadratures are measured instead of the photon properties.

The PNR receiver is fundamentally different from the more commonly used and technically mature homodyne detector. Whether the PNR detector will be the future choice in real-life implementations of binary detectors will depend on the future progress of its technology. A future option is also to use both detector schemes in a receiving station, where the proper detection scheme is chosen according to the currently needed property [49], such as speed, low noise, the capability of performing a full tomography of the state, or the performance for noisy quantum channels [14].

\section{ACKNOWLEDGMENTS}

The work has been supported by the EU project COMPAS and Lundbeckfonden (Grant No. R13-A1274). MT has been supported by MEXT Grant-in-Aid for Young Scientists (B) 22740270.
[1] C. H. Bennett and G. Brassard, in Proceedings of the IEEE International Conference on Computers, Systems, and Signal Processing, Bangalore, India (IEEE, New York, 1984), pp. 175-179.

[2] C. H. Bennett, Phys. Rev. Lett. 68, 3121 (1992).

[3] F. Grosshans et al., Nature (London) 421, 238 (2003).

[4] C. W. Helstrom, Quantum Detection and Estimation Theory (Academic, New York, 1976).

[5] I. Ivanovic, Phys. Lett. A 123, 257 (1987).

[6] D. Dieks, Phys. Lett. A 126, 303 (1988).

[7] A. Peres, Phys. Lett. A 128, 19 (1988).

[8] G. Jaeger et al., Phys. Lett. A 197, 83 (1995).

[9] A. Chefles et al., J. Mod. Opt. 45, 1295 (1998).

[10] J. Fiurásek and M. Jezek, Phys. Rev. A 67, 012321 (2003).

[11] R. S. Kennedy, Research Laboratory of Electronics, MIT, Quarterly Progress Report No. 108, 1973, p. 219 (unpublished).

[12] S. Dolinar, Research Laboratory of Electronics, MIT, Quarterly Progress Report No. 111, 1973, p. 115 (unpublished).
[13] M. Sasaki and O. Hirota, Phys. Rev. A 54, 2728 (1996).

[14] S. Olivares et al., J. Opt. B: Quantum Semiclass. Opt. 6, 69 (2004).

[15] J. M. Geremia, Phys. Rev. A 70, 062303 (2004).

[16] M. Takeoka, M. Sasaki, P. van Loock, and N. Lutkenhaus, Phys. Rev. A 71, 022318 (2005).

[17] M. Takeoka and M. Sasaki, Phys. Rev. A 78, 022320 (2008).

[18] B. Huttner, N. Imoto, N. Gisin, and T. Mor, Phys. Rev. A 51, 1863 (1995).

[19] K. Banaszek, Phys. Lett. A 253, 12 (1999).

[20] R. L. Cook et al., Nature (London) 446, 774 (2007).

[21] C. Wittmann, M. Takeoka, K. N. Cassemiro, M. Sasaki, G. Leuchs, and U. I. Andersen, Phys. Rev. Lett. 101, 210501 (2008).

[22] K. Tsujino, D. Fukuda, G. Fujii, S. Inoue, M. Fujiwara, M. Takeoka, and M. Sasaki, Opt. Express 18, 8107 (2010).

[23] L. Bartušková, A. Cernoch, J. Soubusta, and M. Dusek, Phys. Rev. A 77, 034306 (2008).

[24] C. Wittmann, U. L. Andersen, M. Takeoka, D. Sych, and G. Leuchs, Phys. Rev. Lett. 104, 100505 (2010). 
[25] C. Wittmann et al., J. Mod. Opt. 57, 213 (2010).

[26] C. Silberhorn, T. C. Ralph, N. Lutkenhaus, and G. Leuchs, Phys. Rev. Lett. 89, 167901 (2002).

[27] S. Lorenz et al., Appl. Phys. B 79, 273 (2004).

[28] A. M. Lance, T. Symul, V. Sharma, C. Weedbrook, T. C. Ralph, and P. K. Lam, Phys. Rev. Lett. 95, 180503 (2005).

[29] J. Fiurásek, P. Marek, R. Filip, and R. Schnabel, Phys. Rev. A 75, 050302(R) (2007).

[30] J. Heersink, C. Marquardt, R. Dong, R. Filip, S. Lorenz, G. Leuchs, and U. L. Andersen, Phys. Rev. Lett. 96, 253601 (2006).

[31] R. Dong et al., Nature Phys. 4, 919 (2008).

[32] A. Franzen, B. Hage, J. Di Guglielmo, J. Fiurasek, and R. Schnabel, Phys. Rev. Lett. 97, 150505 (2006).

[33] B. Hage et al., Nature Phys. 4, 915 (2008).

[34] P. Marek and J. Fiurasek, Phys. Rev. A 79, 062321 (2009).

[35] A. M. Lance, H. Jeong, N. B. Grosse, T. Simul, T. C. Ralph, and P. K. Lam, Phys. Rev. A 73, 041801(R) (2006).

[36] A. Ourjoumtsev et al., Nature (London) 448, 784 (2007).
[37] S. A. Babichev, S. Brezger, and A. I. Lvovsky, Phys. Rev. Lett. 92, 047903 (2004).

[38] J. Eisert, S. Scheel, and M. B. Plenio, Phys. Rev. Lett. 89, 137903 (2002).

[39] J. Fiurásek, Phys. Rev. Lett. 89, 137904 (2002).

[40] G. Giedke and J. I. Cirac, Phys. Rev. A 66, 032316 (2002).

[41] D. Walls et al., Quantum Optics (Springer, Berlin, 1994).

[42] A. Ferraro et al., Gaussian States in Continuous Variable Quantum Information (Bibliopolis, Naples, 2005).

[43] K. Banaszek, C. Radzewicz, K. Wodkiewicz, and J. S. Krasinski, Phys. Rev. A 60, 674 (1999).

[44] Z. Ou and H. J. Kimble, Phys. Rev. A 52, 3126 (1995).

[45] B. E. A. Saleh et al., Fundamentals of Photonics, Wiley Series in Pure and Applied Optics (Wiley Interscience, New York, 2007).

[46] A. P. Worsley et al., Opt. Express 17, 4397 (2009).

[47] M. Heid and N. Lutkenhaus, Phys. Rev. A 73, 052316 (2006).

[48] D. Sych et al., e-print arXiv:0902.1895v1 [quant-ph].

[49] B. Qi, Y. Zhao, X. Ma, H. K. Lo, and L. Qian, Phys. Rev. A 75, 052304 (2007) 